\title{
The 45th Annual Meeting of the European Society for Blood and Marrow Transplantation: Quality Management Group - Oral Session
}

C Springer Nature Limited 2019

\author{
24-27 March 2019 Frankfurt, Germany
}

Modified and published with permission from https://www.ebmt.org/annual-meeting

Sponsorship Statement: Publication of this supplement is sponsored by the European Society for Blood and Marrow Transplantation. All content was reviewed and approved by the EBMT Committee, which held full responsibility for the abstract selections.

Presenting author names are bold type in the contributor lists.

\section{3}

Lean management applied at gustave roussy: Improving the process of hematopoietic stem cells freezing and storage

Nigesh Gnaneswaran', Kasthoori Senthilkumar', Florence Aing ${ }^{2}$, Lise Domine ${ }^{3}$, Valentin Aubry', Ulrick Birba $^{1}$, Caroline Gouat ${ }^{1}$, Nathalie Chaput ${ }^{1}$, Valérie Lapierre $^{1}$

1Gustave Roussy, Cell Therapy Centre, Villejuif, France, 2Sup'Biotech, Biotechnology Engineering School, Bioproduction and Quality, Villejuif, France, 3Université Pierre et Marie Curie, Paris, France

Background: Freezing and storage of hematopoietic stem cells is a long and complex process which incorporates the work of different services. In such a process all the services are dependent on each other, so to improve the freezing process of cells in terms of quality and time, the whole system has to be studied. For this a systemic analysis method such as Lean Management is needed. Lean management focuses on identifying and eliminating wastes in terms of quality, delay and cost in a process by studying it as a whole system.

Methods: At Gustave Roussy, patients undergo a blood sampling in the morning prior to the apheresis procedure. Blood samples are analyzed, and the results are validated by the hematological and biological analysis laboratory of the Institute. Then cells are collected by the apheresis staff and transferred to the Processing Facility where the cells are frozen and stored in the appropriate conditions.

The application of lean management is divided in four different phases: 1) field observation of the different processes, 2) description of the observations using different tools, 3) analysis of the observations for identification of wastes and 4) implementation and follow-up of improvement actions.

The observation phase is done by following the different actors during their daily tasks and timing and collecting information through interviews. The most important task was to involve every stakeholder such as the nurses, technicians and doctors in this process.

To describe the different flows (human motion and cell product transport), spaghetti mapping was used. A spaghetti map shows the quantity of motion and transport as flows allowing to identify unnecessary movements.

A Value Stream Map (VSM) was established, representing the different processes we observed by putting them end-to-end. The VSM allows visualizing and analyzing the processes as a whole by representing the different parameters related to each task that were observed such as the time needed to complete a task, the time between two tasks but also the number of operators needed per task.

To analyze the observations, the Ishikawa diagram was used, which allowed identifying the cause of each "waste" in function of its origins (medium, method, manpower, machine and management). The root cause analysis was done by the 5 Whys method. 
Results: More than twenty processes were established on the VSM. Analysis of the flows showed a high number of motion and transport that could be easily reduced by positioning equipment more adequately in the space. The cell product was also waiting in many stages for it to be taken in charge. For example, at the laboratory, the product would wait until the technician dresses up for the clean room. Many wastes in terms of delay were identified during the observations and analysis.

Conclusions: What next? The identified wastes need to be eliminated for process improvement. A LIMS software was put in place allowing a reduction of paper circulation. The next step would be to study small parts of the processes to improve them and to implement a lean approach on a daily basis.

Disclosure: None of the authors has anything to disclose.

\section{4}

Preparing for IEC (CAR-T) Inspection, implementation and implanting into existing quality programme

\section{Valerie Thorpe ${ }^{1,2}$, Robert Wynn ${ }^{1}$, Helen Webster ${ }^{1}$, Joanne Ellis $^{1}$, Kate Owen ${ }^{1}$}

1Royal Manchester Children's Hospital, Paediatric Bone Marrow Transplant, Manchester, United Kingdom, 2Manchester University NHS Foundation Trust, Paediatric Bone Marrow Transplant, Manchester, United Kingdom

Background: Manchester has already treated patients receiving Immune Effector Cell (CAR-T) therapy via research funding and therefore it was vital we achieve accreditation for IEC (CAR-T) following the exciting announcement of NHSE commissioning IEC (CAR-T) therapy to JACIE Accredited Centres. In July 2018 this announcement had a significant impact on quality management and CAR-T Cell Therapy in particular. Selected centres were invited to submit their application and evidence for CAR-T therapy JACIE accreditation; we therefore embarked on another JACIE Inspection experience.

Methods: We followed the standard JACIE process: completion of checklist and submission of all related evidence to meet the standards, mainly surrounding CAR-T; however this did include parts of the already accredited Quality Programme for Royal Manchester Children's Hospital. Several quality meetings took place and the support of the whole team at Manchester was vital to the success of the accreditation. We discussed what we would need i.e. specific SOPs relating to CAR-T, easily recognizable on our already successful QPULSE system and agreed these would be separate from the rest of our SOPS and developed a new section "G" (for gene therapy)! This ensures that all staff can easily locate the SOPs relevant to CAR-T therapy. We updated our Protocol and Quality Management Plan, along with ensuring that the process and procedures we follow in connection with CAR-T therapy were clearly visible and legible for the inspectors to examine. We developed a new CAR-T Flow Chart to outline the process we will follow, which has been added as an appendix to our Quality Manual V8. We looked at our whole Quality Programme and ensured all aspects were up to date as a continuing quality programme for Royal Manchester Children's Hospital.

Results: We were successfully accredited in November 2018, having been inspected in September 2018 and CAR-T is embedded into our overall Quality Programme. We await the decision to be commissioned by NHSE to treat our CAR-T patients in the future.

Conclusions: The Co-ordination and support required for such an inspection requires organisational skills, planning and regular meetings and discussions with the Transplant Team and Quality Management Group members to gather the evidence required specific to CAR-T. The whole Quality Management Team and members of Neurology, PICU and Stem Cell Laboratory are heavily involved in the clinical process, but a central person is required to pull this whole quality management programme together in readiness for Inspection. Positive comments were received from the Inspectors and we were commended on our quality programme. The role of the Quality Manager is vital in ensuring the administration of the programme is upheld.

We will continue to improve our Quality Management Programme going forward and make the necessary changes as and when required to meet the ever changing needs of the NHS and the Transplant Service as a whole for our patients, who are at the heart of everything we do.

Disclosure: Nothing to declare

\section{5}

Oral anticancer medication adherence of migrants and ethnic minorities with haematological malignancies, a challenge for EBMT?

Sandra Michiels ${ }^{1,2,3}$, Dominique Bron ${ }^{1}$, Patrick Crombez, Marie Vercruyssen ${ }^{1}$, Sandra Tricas-Sauras ${ }^{3}$, Fati Kirakoya ${ }^{2}$

1Institut Jules Bordet, Brussels, Belgium, 2Université Libre de Bruxelles, School of Public Health - Center for Research in Epidemiology, Biostatistics, and Clinical Research, Brussels, Belgium, 3Université Libre de Bruxelles, School 
of Public Health - Center for Research in Social Approaches to Health, Brussels, Belgium

Background: Migration is increasing worldwide, with accumulating evidence of disparities. Migrants and Ethnic Minorities (MEMs) are identified as one of the most vulnerable group in health provision. In the context of haematological cancer patients (HECPs), significant "racial" and ethnic disparities have been reported regarding incidence and survival rates. At the same time, HECPs are increasingly treated by Oral Anticancer Medications (OAMs), including for bridging to transplant, and oncohaematological teams have to deal with the survey of adherence. These two evolutions raise the question of MEMs' adherence and their integration in cancer adherence studies.

Methods: We conducted a scoping review of scientific literature regarding adherence to treatment in MEMs patients, particularly to OAMs.

Results: Data are still scarce regarding how cancer patients adhere to their medication plan, particularly for those with malignancies other than breast cancer.

In HECPs, research has shown that poor adherence impacts the quality of response and the risk of relapse.

Nowadays, adherence is understood as the complex interplay between predisposing factors, patients' knowledge and beliefs, and interactions between patients and clinicians.

Recent findings underline differences and difficulties between physician and patient coming from different cultural and/or ethnic backgrounds. Little is known about miscommunication causes. This is not only a language issue but it is also intimately related to cultural differences in the way people think about health, disease and care.

It would seem therefore reasonable to anticipate higher risk of poor adherence among MEMs in foreign context. Unfortunately, this population group seems to have been forgotten from adherence studies in oncology.
If "race" or ethnicity have been frequently reported to be a predictor of poor adherence, these studies entail important limitations which prevent definite conclusions. Beyond the fact that most research studies concern other chronic diseases, they are mainly conducted in USA and include socioeconomic factors such as uninsured driving that may interact with ethnicity in explaining nonadherence prevalence. Most adherence studies do not report patients' ethnic background either. In general, as for other cancer clinical trials, MEMs appear particularly underrepresented $(2-11 \%)$.

Conclusions: The results of this scoping review highlight important gaps in knowledge and research concerning MEMs' adherence.

Their underrepresentation in adherence studies risks the generalizability of results in the context of diverse minority populations.

Additionally, geographical concentration of data in Anglo-Saxon countries and regarding other chronic diseases raises the question of results' applicability to HECPs and for other countries.

With the emergence of novel oral therapeutics in oncohaematology it becomes urgent to identify differences in access, use and quality of care that may lead to disparities in health outcomes. As an expert association, EBMT can play a key role in answering these unmet needs. EBMT should recognize existing disparities and contribute to the development of guidelines supporting the inclusion of MEMs and other underrepresented populations in oncohaematology adherence studies. By supporting extensive research EBMT would contribute to provide urgent missing data about how MEMs with haematological malignancies adhere and persist to their OAM in our European contexts and to develop consistent strategies.

Clinical Trial Registry: NA

Disclosure: No disclosure 